\title{
Hinode EUV spectroscopic observations of coronal oscillations
}

\author{
R. Erdélyi and Y. Taroyan
}

\author{
$\mathrm{SP}^{2} \mathrm{RC}$, Department of Applied Mathematics, University of Sheffield, Sheffield, S3 7RH, UK \\ e-mail: [robertus;y.taroyan]@sheffield.ac.uk
}

Received 26 May 2008 / Accepted 25 August 2008

\section{ABSTRACT}

\begin{abstract}
Context. Waves offer a unique opportunity to understand and diagnose the properties of the solar coronal plasma. Aims. Hinode/EIS observations are analysed to detect wave and oscillatory motions in the solar corona. Methods. The EIS observations were carried out using a selection of EUV lines. Hinode/XRT images are taken concurrently. Results. Two examples of oscillations in active regions are presented. We analysed the evolution of the intensities and Doppler shifts. The Hinode/XRT images suggest that both events occurred along loop-like structures. The first event is interpreted as a slow sausage (acoustic) type wave with a period of $1.2 \mathrm{mHz}$. The second example is associated with a transverse, most likely kink type, wave with a period of $3 \mathrm{mHz}$. The EUV line ratios were then used to determine the value of the coronal magnetic field.
\end{abstract}

Key words. techniques: spectroscopic - Sun: corona - Sun: oscillations - line: profiles - magnetohydrodynamics (MHD)

\section{Introduction}

Magnetohydrodynamic (MHD) waves are important both energetically and as a diagnostic tool in solar atmospheric research. Determining the physical parameters of the solar coronal plasma is important for understanding such fundamental processes as the heating of the coronal plasma and acceleration of the solar wind. In the past decade, new-generation satellites such as SoHO and TRACE have provided evidence of various types of MHD waves. Solar coronal structures support both propagating and standing MHD waves. Propagating slow magnetoacoustic waves have been detected in coronal plumes (Ofman et al. 1997, 1999) and near the footpoints of coronal loops (Berghmans \& Clette 1999; De Moortel et al. 2000). Williams et al. (2001) have studied fast magnetoacoustic waves propagating along a loop. Among the standing, fast MHD waves observed in coronal loops are the global kink (Aschwanden et al. 1999; Nakariakov et al. 1999) and sausage-mode oscillations (Nakariakov et al. 2003). Oscillations interpreted as standing, slow magnetoacoustic waves have been studied in hot $(T>6 \mathrm{MK})$ loops with the SUMER spectrometer on board the SoHO satellite (Wang et al. 2002).

The Hinode satellite, launched in 2006, offers new opportunities for studying the Sun's atmosphere. The mission is equipped with several instruments that complement one another. The present letter reports the detection of magnetoacoustic oscillations by the EUV Imaging Spectrometer (EIS). Two examples are presented. The first event occurred on 2007 February 19 and the second on 2007 February 21. The EIS observations were carried out in a sit-and-stare spectroscopy mode using a selection of 17 different EUV lines. Both events are best seen in the strong Fe XII $195 \AA$ A line. Concurrent Hinode/XRT images show the location of the EIS slit on the solar disc. The images suggest that, in both cases, the oscillations occur in loop-like structures at different locations. We discuss the nature and the origin of these oscillatory motions.

\section{Observations}

The EUV imaging-spectrometer Hinode/EIS has two CCDs each covering a 40 A wavelength range: $170-210 \AA$ and 250-290 A. The wavelength response of EIS has two peaks at around $195 \AA$ and $271 \AA$ corresponding to the two CCDs. The EIS has both narrow ( $1^{\prime \prime}$ and $2^{\prime \prime}$ wide) slits, and wider (40" and 266") imaging slots, all with up to 512" in the solar_Y direction. It is able to make slit observations of active regions in ten seconds and of the quiet Sun in between thirty and sixty seconds, and of flares in approximately one second. The spectral resolution can be less than $1 \mathrm{~km} \mathrm{~s}^{-1}$ for the Doppler shift. More details of the Hinode/EIS characteristics are given by Culhane et al. (2007) and Kosugi et al. (2007). Below, two examples of oscillations are presented.

\subsection{February 19, 2007}

The 2007, February 19 observations are sit-and-stare observations in which a $1^{\prime \prime}$ wide EIS slit with a window height of 512" is used. The observations began at 21:06 UT and lasted for about an hour. The location of the slit is marked by a vertical line in the XRT image (Fig. 1) and in the EIS 40" slot images taken after 22:10 UT (Fig. 2). The exposure time was around $30 \mathrm{~s}$ and the total number of exposures 110 . The centre of the slit has average coordinates solar_X $=6^{\prime \prime}$, solar_Y $=-35^{\prime \prime}$. Standard procedures for EIS data reduction were applied to include calibrating the data and creating error arrays, applying Gaussian fitting to each line profile and correcting for the slit tilt and orbital variation. The resulting intensities and Doppler shifts were measured in DN (data number) and in $\mathrm{km} \mathrm{s}^{-1}$. The Doppler shift has an error of less than $1 \mathrm{~km} \mathrm{~s}^{-1}$.

Oscillatory behaviour was detected between pixels 230 and 234. These pixels are marked in the XRT and EIS images (Figs. 1 and 2). In order to verify that the oscillations are not an instrumental artifact, the Doppler-shift time series along the 


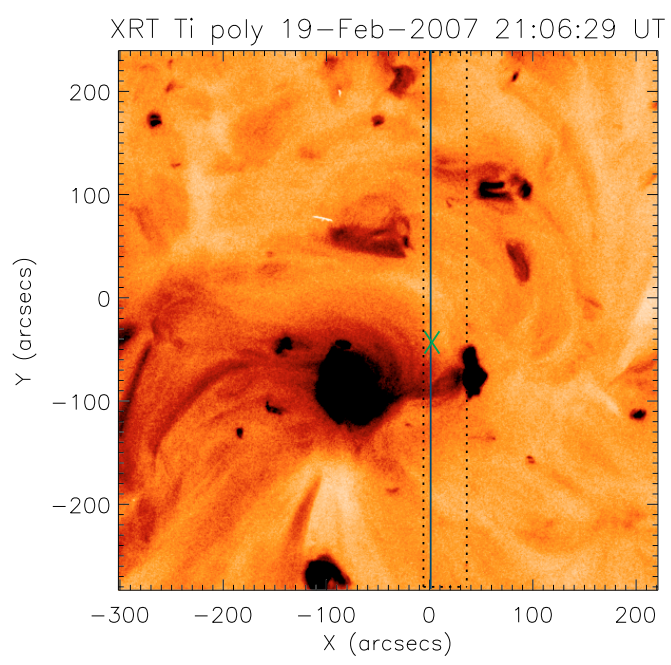

Fig. 1. An XRT image of the solar disc taken 2007 February 19, 2007. The dotted rectangle shows the region of interest in which EIS imaging/spectroscopic observations were carried out. The $1^{\prime \prime}$ EIS slit is marked by a vertical line. The cross indicates the location of the pixels that registered the oscillations starting at 21:06 UT.
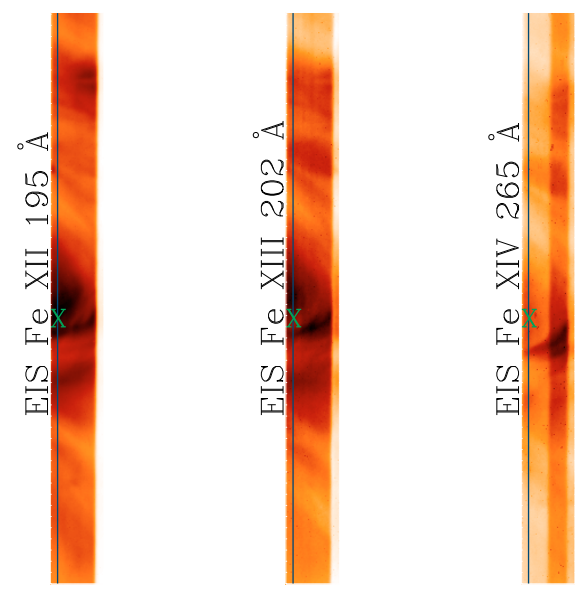

Fig. 2. EIS 40" slot observations lasting from 22:10 to 22:35 UT. Each image covers a $40^{\prime \prime} \times 512^{\prime \prime}$ rectangular area that is marked by a dotted line in Fig. 1. The 1" EIS slit is marked by a vertical line. The cross indicates the location of the pixels that show oscillations.

entire slit is examined (Fig. 3). Except for the narrow horizontal stripe, no significant wave power was found elsewhere along the slit. An average of the intensity and the Doppler shift taken over these pixels for Fe XII $195 \AA$, Fe X $184.7 \AA$, and Fe XIII $202 \AA$ is plotted in Fig. 4. The oscillations show up both in the intensity and in the Doppler shift, which appear to be phase-shifted by a quarter period as shown by the sinusoidal fit. The maximum amplitude for the Doppler shift is about $4 \mathrm{~km} \mathrm{~s}^{-1}$.

We also applied wavelet analysis, and details of the procedure are given by Torrence \& Compo (1998). For the convolution of the time series the Morlet function was chosen, and to establish whether the oscillations are real, a randomization method was implemented to estimate the significance level of the peaks in the wavelet spectrum. The top panel in Fig. 5 shows the evolutions of the Doppler shift in spectral pixels averaged over $5^{\prime \prime}$ in solar_Y. The mean value has been subtracted. The bottom left panel shows the wavelet spectrum and the right panel shows the global wavelet spectrum, which is the sum of the wavelet power

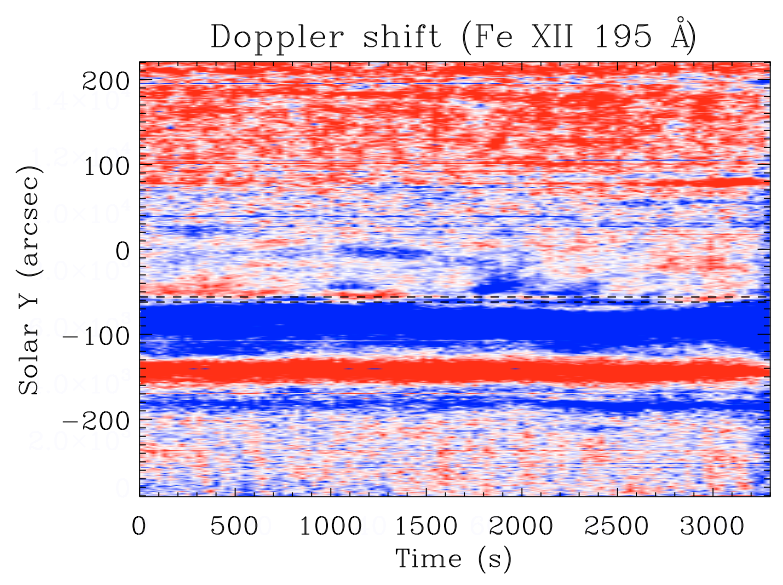

Fig. 3. Doppler-shift time series along the 1" EIS slit for the observations starting at 21:06 UT on 2007 February 19. Positive and negative Doppler shifts are represented by red and blue colours, respectively. The selected line is Fe XII $195 \AA$. The exposure time is $30 \mathrm{~s}$ and the total number of exposures 110 . The narrow stripe between the horizontal dashed lines marks the pixels with high oscillation power and corresponds to the cross in Figs. 1 and 2.
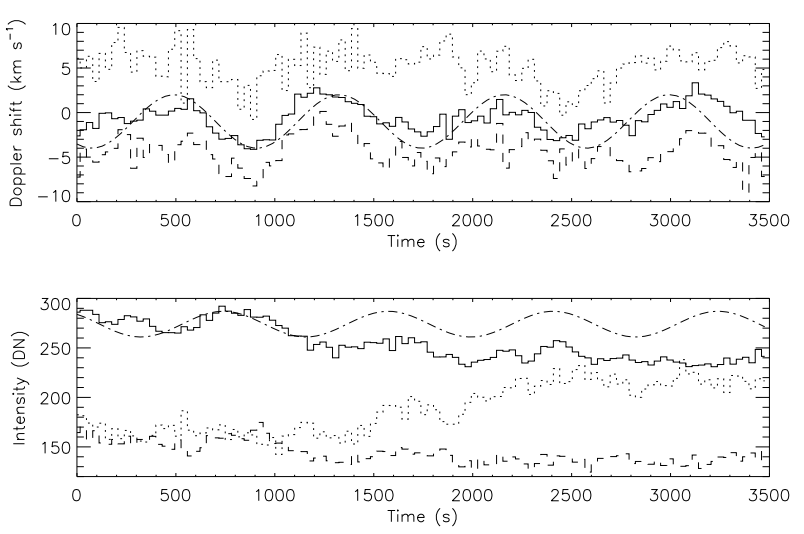

Fig. 4. The Doppler shift and intensity time series averaged over five pixels between the dashed lines in Fig. 3. The solid, dotted, and dashed curves correspond to Fe XII $195 \AA$ A, Fe X $184.7 \AA$, and Fe XIII $202 \AA$, respectively. The quarter-period phase shifted dashed-dotted curves represent sinusoidal fits to the Doppler shift and intensity oscillations.

over time at each oscillation period. Cross-hatched regions indicate the cone of influence where edge effects become strong.

Due to the limited temporal resolution, only frequencies less than $10 \mathrm{mHz}$ are considered. The wavelet for the Doppler shift (Fig. 5) shows strong power at $1.2 \mathrm{mHz}$ for almost the entire duration of the observations. The corresponding power in the global wavelet has a significance level above 99\%. In Fig. 6 a similar wavelet analysis procedure is applied to the intensity. A linear fit was subtracted from the time series to remove the background trend. The peak at $1.3 \mathrm{mHz}$ is above the $99 \%$ significance level. The peak for the intensity has a slightly higher frequency that could be due to the phase shifts it may suffer as predicted by Taroyan \& Bradshaw (2008). The XRT and EIS images (Figs. 1 and 2) suggest that the oscillations correspond to a footpoint region of a loop where the slit crosses the loop. According to linear MHD wave theory, intensity and Doppler shift oscillations are usually associated with an acoustic longitudinal wave. From the analysis of the XRT image, longitudinal motions at the marked location should have a line-of-sight component resulting in the observed Doppler shift. The XRT 


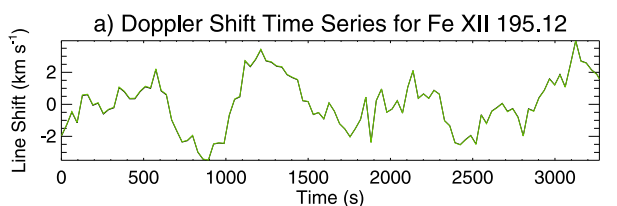

WAVELET ANALYSIS
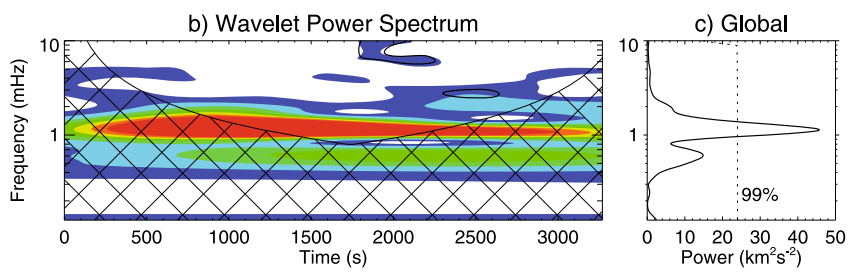

Fig. 5. Wavelet analysis for the Fe XII $195 \AA$ Doppler-shift time series shown in Fig. 3. The top panel a) shows the Doppler-shift time series with the subtracted mean value. The bottom left panel $\mathbf{b})$ displays the wavelet power spectrum. The red colour represents high power, and the blue colour corresponds to low power. The bottom right panel $\mathbf{c}$ ) is the global wavelet spectrum. In the global wavelet diagram, the dotted lines indicate a $99 \%$ significance level.

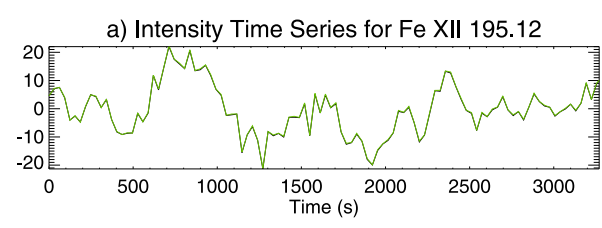

WAVELET ANALYSIS
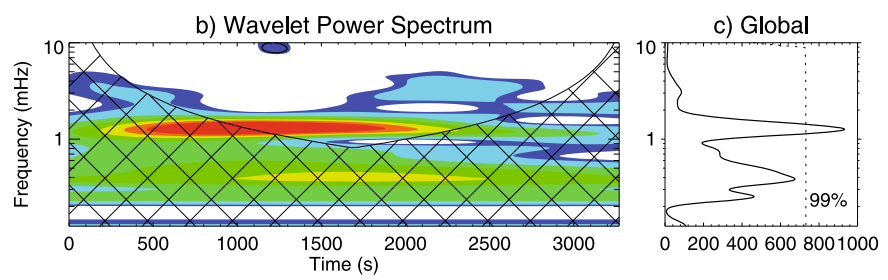

Fig. 6. The same as in Fig. 5 but applied to the Fe XII $195 \AA$ Antensity time series. A linear fit has been subtracted from the original time series to remove the background trend.

movie associated with the EIS observations shows that the oscillations are preceded by a small microflare near the footpoint, which would heat the plasma and trigger the oscillations. Such events have been observed by SUMER in high-temperature lines (Wang et al. 2003, 2005). The microflare brightening starts at 20:30 UT and ends at 21:00 UT, i.e., around the time when the oscillation starts. The details and the forward modelling of microflare-triggered oscillations are discussed by Taroyan et al. $(2005,2007)$. The intensity increase seen in lower tempereature lines (e.g., Fe VIII and the decrease in higher temperature lines (e.g., Fe XII, Fe XIII, Ca XIII) conforms with the cooling scenario following a transient heat deposition. Fundamental mode standing sausage waves have so far been detected only in hot ( $T>6 \mathrm{MK}$ ) loops (Wang et al. 2002). The presented example could be the first proof of the existence of such oscillations in cooler loops. The rather slow damping is more obvious in the intensity than in the Doppler shift oscillations and requires further theoretical investigation. The quarter-period phase shift seen between the intensity and Doppler shift oscillations in Fig. 4 and their relative amplitudes also conform well with a standing-wave scenario (Taroyan \& Bradshaw 2008).

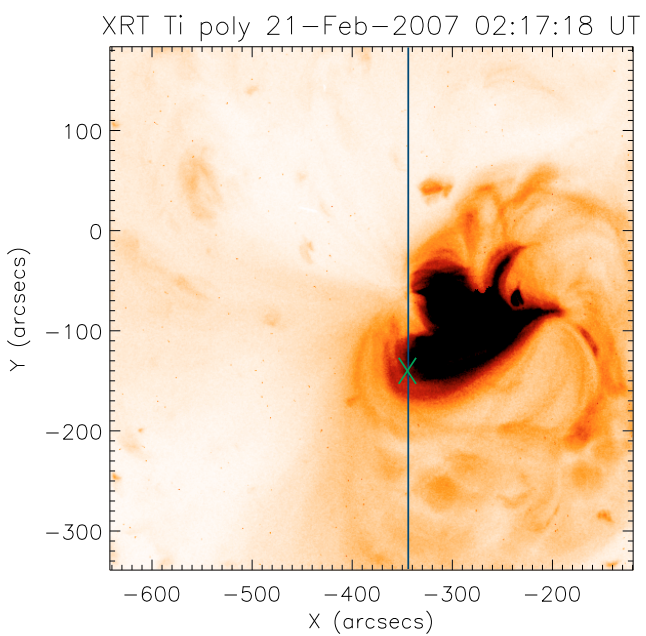

Fig. 7. Oscillating coronal loops observed by XRT on the solar disc. The $1^{\prime \prime}$ EIS slit is marked by a vertical line. The cross indicates the location of the pixels that show oscillations starting at 02:18 UT 2007 February 21.
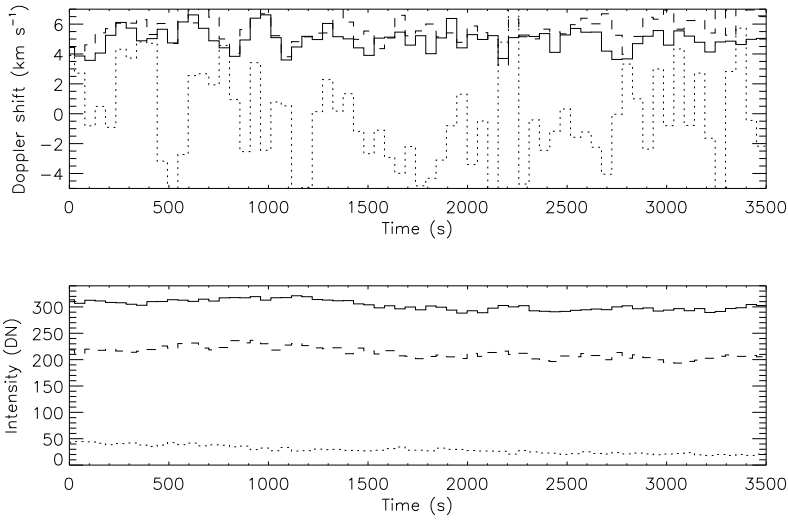

Fig. 8. Intensity (DN) and Doppler-shift $\left(\mathrm{km} \mathrm{s}^{-1}\right)$ time series for Fe XII $195 \AA$ Fe VIII $185 \AA$ and Fe XIII $202 \AA$ are represented by the solid, dotted, and dashed curves. The observations start at 02:18 UT 2007 February 21. An average over 5 adjacent pixels is taken along the slit. The exposure time is $50 \mathrm{~s}$ and the total number of exposures 70 .

\subsection{February 21,2007}

The 2007, February 21 observations are again sit-and-stare observations with the $1^{\prime \prime} \times 512^{\prime \prime}$ EIS slit. The exposure time is $50 \mathrm{~s}$ and the total number of exposures is 70 . The observations started at 02:18 UT and lasted about an hour. The observations were carried out in 17 different lines as in the previous case. During the observations, the centre of the slit had average coordinates solar_X $=-348^{\prime \prime}$, solar_Y $=-91^{\prime \prime}$. The XRT image shows the position of the slit (Fig. 7). The standard procedure for data reduction was applied. The Doppler shift has an error of less than $0.6 \mathrm{~km} \mathrm{~s}^{-1}$ due to the longer exposure time. Oscillatory behaviour was detected between pixels 200 and 204. An average of the Doppler shift and intensity was taken over these pixels for Fe XII $195.12 \AA$, Fe VIII $185 \AA$, Fe XIII $202 \AA$, and plotted in Fig. 8. A damped oscillation with a maximum amplitude of $1.5 \mathrm{~km} \mathrm{~s}^{-1}$ can be seen in the Doppler shift. The intensity in all three lines remains almost constant and does not exhibit any oscillatory behaviour. Similar to the previous case, no significant wave power is found elsewhere along the slit.

The wavelet for the Doppler shift (Fig. 9) show strong oscillations at $3 \mathrm{mHz}$ that gradually decay. The corresponding 


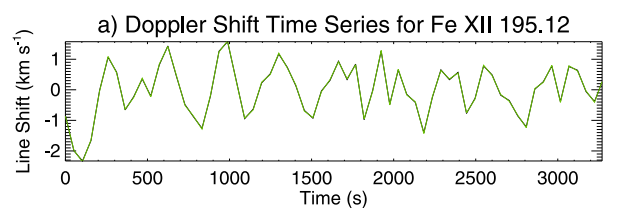

WAVELET ANALYSIS

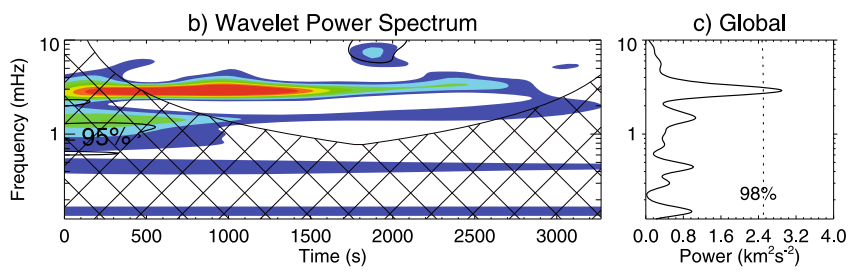

Fig. 9. Wavelet analysis for the Fe XII $195 \AA$ A Doppler-shift time series shown in Fig. 7. The top panel a) shows the Doppler-shift time series with the subtracted mean value. The bottom left panel b) displays the wavelet power spectrum. The red colour represents high power, and the blue colour corresponds to low power. The bottom right panel c) is the global wavelet spectrum. In the global wavelet diagram, the dotted lines indicate $98 \%$ significance level.

power in the global wavelet has a significance level above $98 \%$. The XRT snapshot indicates that the pixels showing oscillations correspond to a near apex region of a loop where the slit crosses the loop. There is no evidence of an off-set micro-flare or an intensity explosion with a subsequent decrease. Also the high frequency and the absence of intensity oscillations do not favour any interpretation in terms of a slow mode. The XRT image suggests that transverse motions at the marked location should have a line-of-sight component resulting in the observed Doppler shift. Doppler-shift oscillations without accompanying intensity oscillations would indicate the presence of either a torsional Alfvén wave or a magnetoacoustic kink wave. However, a more plausible explanation is the kink wave since the sum of the torsional motions taken along the slit would most likely cancel out the oscillations seen in the Doppler shift (or, at least, the oscillations would not be in-phase in different pixels). Usually the excitation of transverse kink oscillations is associated with flares and erupting prominences. The present example seems to have a different origin that we currently do not understand. On the other hand, the five-minute period is similar to the periods of previously reported kink oscillations observed by TRACE. Kink oscillations have a broad range of damping times (Aschwanden et al. 2002). The present example is characterised by rather weak damping.

Estimates of coronal magnetic fields are one important application of wave studies. In the case of fundamental mode fast kink oscillations, the magnetic field strength is given by the formula

$B_{0}=7.9 \times 10^{-13} \frac{n_{\mathrm{i}}^{1 / 2} d \sqrt{1+n_{\mathrm{e}} / n_{\mathrm{i}}}}{P}$

where $n_{\mathrm{e}}$ and $n_{\mathrm{i}}$ are the external and internal number densities measured in $\mathrm{m}^{-3}, d$ is the loop length measured in $\mathrm{m}$, and $P$ the oscillation period (Nakariakov \& Ofman 2001). The XRT image suggests that the loop length is between $0.8-1.2 \times 10^{8} \mathrm{~m}$, assuming that the loop has a semicircular shape. We also assume that the measured period of $300 \mathrm{~s}$ represents the period of the fundamental mode as the most natural and easiest to excite. The density is measured using the ratio between the Fe XII 186.88 and Fe XII 195.12 line intensities. The derived value of the density varies between $4 \times 10^{14} \mathrm{~m}^{-3}$ and $8 \times 10^{14} \mathrm{~m}^{-3}$. The resulting magnetic field strength of the oscillating loop is $B_{0}=10 \pm 6 \mathrm{G}$.

\section{Summary}

The present letter reports on the first detection of coronal oscillations by Hinode/EIS. The observations were carried out in a sit-and-stare 1" spectroscopy mode. The intensity and Doppler-shift time series were analysed to interpret the nature of the oscillations. The concurrent XRT images show the location of the oscillations on the solar disc. The first example (2007 February 19) consists of both intensity and Doppler-shift oscillations. These are interpreted as longitudinal sausage waves along a loop that are excited by a footpoint microflare. The second event (2007 February 21) has no intensity component in the oscillations. It is most likely a kink type transverse wave. TRACE observations suggest that kink oscillations are usually triggered by flares and prominence eruptions. No such events are seen in the present example, and the source of the oscillations currently remains unknown. Finally, we note that there are other examples that have not been included in this Letter. A more comprehensive analysis including modelling aspects will be presented in a full forthcoming study.

Acknowledgements. R.E. acknowledges M. Kéray for patient encouragement, NSF, Hungary (OTKA, K67746), and STFC. Y.T. is grateful to the Leverhulme trust for financial support. The authors also thank the referee for the constructive comments and suggestions.

\section{References}

Aschwanden, M. J., Fletcher, L., Schrijver, C. J., \& Alexander, D. 1999, ApJ, 520,880

Aschwanden, M. J., De Pontieu, B., Schrijver, C. J., \& Title, A. M. 2002, Sol. Phys., 206, 99

Berghmans, D., \& Clette, F. 1999, Sol. Phys., 186, 207

Culhane, J. L., Harra, L. K., James, A. M., et al. 2007, Sol. Phys., 243, 19

De Moortel, I., Ireland, J., \& Walsh, R. W. 2000, A\&A, 355, L23

Kosugi, T., Matsuzaki, K., Sakao, T., et al. 2007, Sol. Phys., 243, 3

Nakariakov, V. M., \& Ofman, L. 2001, A\&A, 372, L53

Nakariakov, V. M., Ofman, L., DeLuca, E. E., Roberts, B., \& Davila, J. M. 1999, Science, 285, 862

Nakariakov, V. M., Melnikov, V. F., \& Reznikova, V. E. 2003, A\&A, 412, L7

Ofman, L., Rokoli, M., Poletto, G., Noci, C., \& Kohl, J. L. 1997, ApJ, 491, L111

Ofman, L., Nakariakov, V. M., \& DeForest, C. E. 1999, ApJ, 514, 441

Taroyan, Y., \& Bradshaw, S. J. 2008, A\&A, 481, 247

Taroyan, Y., Erdélyi, R., Doyle, J. G., \& Bradshaw, S. J. 2005, A\&A, 438, 713

Taroyan, Y., Erdélyi, R., Wang, T. J., \& Bradshaw, S. J. 2007, ApJ, 659, L173

Torrence, C., \& Compo, G. P. 1998, Bull. Meteor. Soc., 79, 61

Wang, T. J., Solanki, S. K., Curdt, W., et al. 2002, ApJ, 574, L101

Wang, T. J., Solanki, S. K., Curdt, W., et al. 2003, A\&A, 406, 1105

Wang, T. J., Solanki, S. K., Innes, D. E., et al. 2005, A\&A, 435, 753

Williams, D. R., Phillips, K. J. H., Rudawy, P., et al. 2001, MNRAS, 326, 428 